\title{
Unobtrusive Multimodal Biometric Authentication: The HUMABIO Project Concept
}

\author{
loannis G. Damousis, ${ }^{1}$ Dimitrios Tzovaras, ${ }^{1}$ and Evangelos Bekiaris ${ }^{2}$ \\ ${ }^{1}$ Informatics and Telematics Institute of the Center for Research and Technology Hellas, 57001 Thermi-Thessaloniki, Greece \\ ${ }^{2}$ Hellenic Institute of Transport of the Center for Research and Technology Hellas, 57001 Thermi-Thessaloniki, Greece
}

Correspondence should be addressed to Ioannis G. Damousis, damousis@iti.gr

Received 10 May 2007; Revised 27 August 2007; Accepted 25 November 2007

Recommended by Konstantinos N. Plataniotis

\begin{abstract}
Human Monitoring and Authentication using Biodynamic Indicators and Behavioural Analysis (HUMABIO) (2007) is an EU Specific Targeted Research Project (STREP) where new types of biometrics are combined with state-of-the-art sensorial technologies in order to enhance security in a wide spectrum of applications. The project aims to develop a modular, robust, multimodal biometrics security authentication and monitoring system which utilizes a biodynamic physiological profile, unique for each individual, and advancements of the state of the art in behavioural and other biometrics, such as face, speech, gait recognition, and seat-based anthropometrics. Several shortcomings in biometric authentication will be addressed in the course of HUMABIO which will provide the basis for improving existing sensors, develop new algorithms, and design applications, towards creating new, unobtrusive biometric authentication procedures in security sensitive, controlled environments. This paper presents the concept of this project, describes its unobtrusive authentication demonstrator, and reports some preliminary results.
\end{abstract}

Copyright (c) 2008 Ioannis G. Damousis et al. This is an open access article distributed under the Creative Commons Attribution License, which permits unrestricted use, distribution, and reproduction in any medium, provided the original work is properly cited.

\section{INTRODUCTION}

Biometrics measure unique physical or behavioural characteristics of individuals as a means to recognize or authenticate their identity. Common physical biometrics include fingerprints, hand or palm geometry, and retina, iris, or facial characteristics. Behavioural characteristics include signature, voice (which also has a physical component), keystroke pattern, and gait. Although some technologies have gained more acceptance than others, it is beyond doubt that the field of access control and biometrics as a whole shows great potential for use in end user segments, such as airports, stadiums, defense installations, and the industry and corporate workplaces where security and privacy are required.

A shortcoming of biometric security systems is the discrimination of groups of people whose biometrics cannot be recorded well for the creation of the reference database, for example, people whose fingerprints do not print well or they even miss the required feature. These people are de facto excluded by the system. In that respect, the research on new biometrics that exploit physiological features that exist in every human (such as electroencephalogram (EEG) and elec- trocardiogram (ECG) features), thus rendering them to be applicable to the greatest possible percentage of the population, becomes very important.

Since authentication takes place usually only once, identity fraud is possible. An attacker may bypass the biometrics authentication system and continue undisturbed. A cracked or stolen biometric system presents a difficult problem. Unlike passwords or smart cards, that can be changed or reissued, absent serious medical intervention, a fingerprint or an iris is forever. Once an attacker has successfully forged those characteristics, the end user must be excluded from the system entirely, raising the possibility of enormous security risks and reimplementation costs. Static physical characteristics can be digitally duplicated, for example, the face could be copied using a photograph, a voice print using a voice recording, and the fingerprint using various forging methods. In addition, static biometrics could be intolerant of changes in physiology such as daily voice changes or appearance changes. Physiological dynamic indicators could address these issues and enhance the reliability and robustness of biometric authentication systems when used in conjunction with the usual biometric techniques. The nature of 
these physiological features allows the continuous authentication of a person (in the controlled environment), thus presenting a greater challenge to the potential attacker.

Another problem that current biometric authentication solutions face is the verification of the subject's aliveness. Spoofing attacks to biometric systems usually utilize artificially made features such as fingerprints, photographs, and others, depending on the feature the system uses for authentication $[1,2]$. In order to cope with this situation, extensive research takes place in order to create aliveness checks custom tailored to each biometric parameter. Some of these solutions work better than the others; however aliveness check remains a difficult task. Due to the nature of biodynamic indicators that describe a person's internal physiology, an authentication system that utilizes them performs a de facto and reliably an aliveness check of that person.

The identity theft scenario is especially true for biometric systems that are based solely on a single biometric feature, namely, unimodal biometrics. This kind of biometric systems may not always meet performance requirements; they may exclude large numbers of people and are vulnerable to everyday changes and lesions of the biometric feature. Because of this, the development of systems that integrate two or more biometrics is emerging as a trend. Experimental results have demonstrated that the identities established by systems that use more than one biometric could be more reliable and applicable to large population sectors, and improve response time $[3,4]$.

Finally, a major shortcoming of all biometrics is the obtrusive process for obtaining the biometric feature. The subject has to stop, go through a specific measurement procedure, which depends on the biometric that can be very obtrusive, wait for a period of time, and get clearance after authentication is positive. Emerging biometrics such as gait recognition and technologies such as automated person/face detection can potentially allow the nonstop (on-the-move) authentication or even identification which is unobtrusive and transparent to the subject and become part of an ambient intelligence environment. These biometrics and technologies however are still in research phase and even though the results are promising, they have not yet led to products or their market share is minimal.

\section{HUMABIO CONCEPT}

HUMABIO is a research and development project that aims to enhance security at supervised and controlled environments. The project research revolves around two main axes.

\subsection{The biometric authentication enhancement via the use of new types of biometrics that describe the internal physiology of the subject, their cooperation with existing behavioural biometrics, and the improvement of widely used system solutions}

\subsubsection{Internal physiology biometrics}

HUMABIO explores the use of physiological modalities that, contrary to commonly used biometrics, describe the internal physiology of a person and they either have never been used in the past or are still in research phase that has not led to conclusive or exploitable results due to the limited number of subjects participating in the research or the technical and user acceptance restrictions imposed by the existing measuring means, respectively [5-9].

By investigating the authenticating capacity of biodynamic indicators such as event related potentials (ERP) [8, 9], EEG baseline [6,7] and heart dynamics [5] and implementing the ones that show strong potential into the final system, HUMABIO aims to overcome several of the shortcomings of the current biometric solutions.

Specifically,

(1) it can be applied to the totality of the population since these features exist in everyone;

(2) biodynamic indicators ensure the aliveness of the individual, and the measurements take place in a nonintrusive way, for example, in contrast to DNA biometrics;

(3) spoofing is minimized in two ways: the aliveness check which is inherited in the biodynamic indicators and the synchronous use of multiple biometrics;

(4) finally, HUMABIO biometric features allow the continuous authentication and monitoring of the individual in a controlled environment, decreasing further the possibility of spoofing.

The use of these novel biometrics will also enable HUMABIO system to act as a monitoring system $[10,11]$ that validates the normal emotional and physiological state of employees and operators and guarantees the proper execution of critical and sensitive tasks that involve risks to the environment and the people.

\subsubsection{External physiology and behavioural biometrics}

In order to increase the reliability and the applicability of the HUMABIO system, external physiology and behavioural biometrics are also utilized. Based on criteria such as unobtrusiveness level, maturity of the technology, and biometric capacity, face, voice, and gait recognition biometrics were selected to be included in the HUMABIO system and complement the biometrics that describe the person's internal physiology. In addition, a new biometric is introduced: authentication via the extraction of the anthropometric profile using a sensing seat.

\subsection{The improvement of security and safety through the minimization of human operator related accidents in critical operations}

This is accomplished through research on algorithms and systems that guarantee the capacity of the individual to perform his or her task before and during the execution of the task. In that way, the system proposes three main operation phases that are indicated in Table 1.

The authentication phase is characterized by three states according to the application scenario. The initial authentication takes place when the subject logs into the protected 


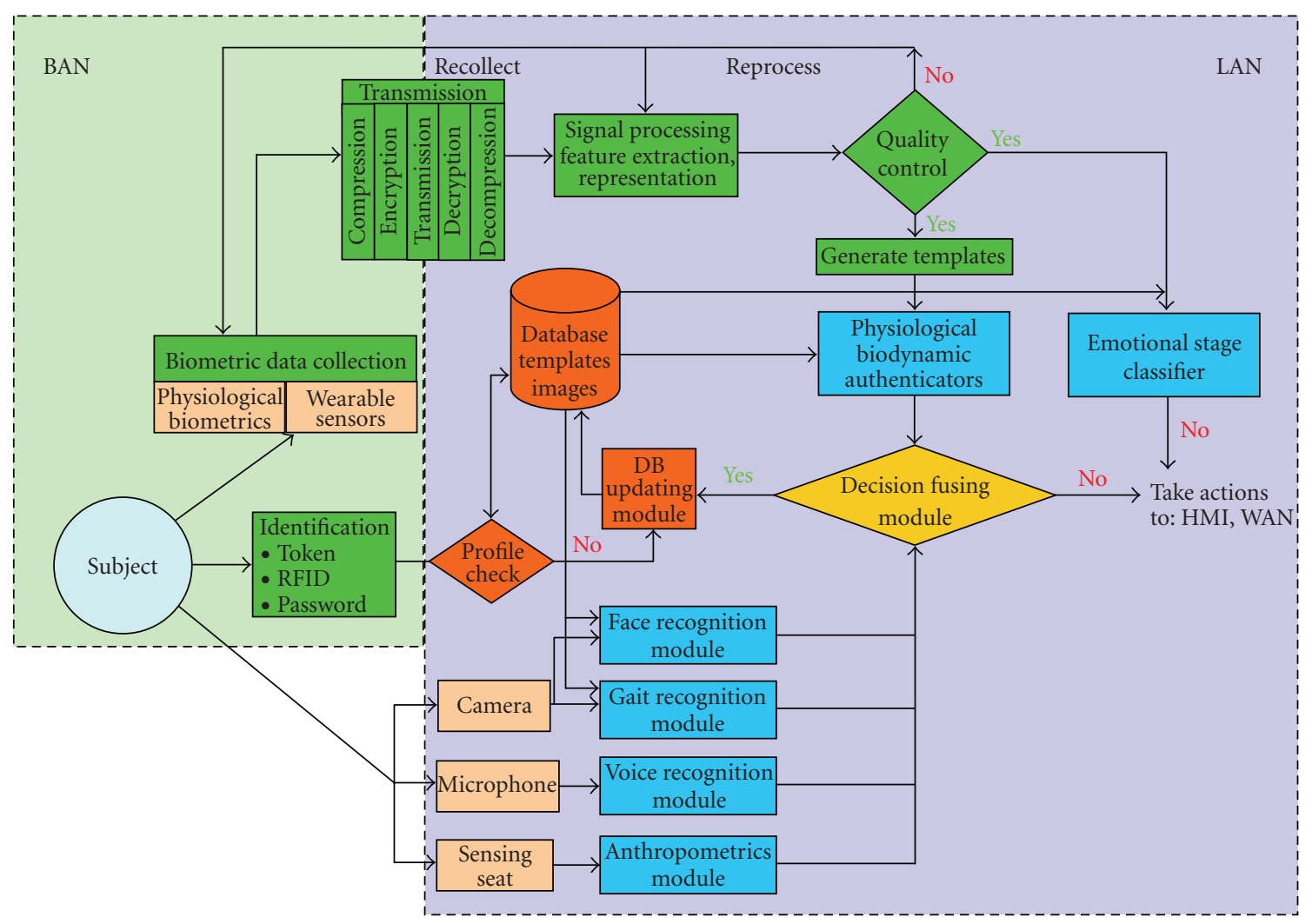

FIgURE 1: HUMABIO architecture concept.

system. This is typically the same process that is being used in all security systems: the subjects declare their identity using a login or a token in order to gain access to a resource and then a password is used to authenticate his/her identity. In the context of HUMABIO, the initial authentication process will be enhanced and will also include face recognition, text dependent voice analysis, and innovatory EEG authentication based on the analysis of event related potentials that are registered on the scalp.

The continuous authentication state is an innovation of HUMABIO that enhances the security of fixed place workstations by reducing the possibility of system spoofing. The subject's EEG, ECG, and other physiological features that show intrapersonal stability and can act as biological signatures are continuously monitored in order to guarantee the identity of the operator throughout the whole process. Face and speaker authentication will be utilized in parallel to improve the reliability of the system.

Nonobtrusive authentication will be implemented in the context of HUMABIO in order to widen the applicability of the system. It involves automatic authentication of authorized personnel that can move freely in restricted areas. The authentication of an individual that carries an ID in the form of radio frequency identification (RFID) card will take place, using face and gait recognition techniques in order to minimize the obtrusiveness and maximize the convenience from the subject's point of view. EEG and other physiological fea- tures could be used in this scenario depending on the user's requirements and the obtrusiveness level of the sensors that will collect the physiological data.

The validation phase of the subjects' initial "nominal" state will guarantee that the subjects have the capacity to perform their tasks. This process will be rather obtrusive since it will use ERP and body sway methods and will last several minutes. The aim is to detect possible deficiencies (deriving from drug consumption, sleep deprivation, etc.) through measurements of features that can describe the person's state. This phase will be applied to critical operation scenarios that require the operator's full attention and readiness such as professional driving or air traffic controlling.

The monitoring phase is the generalization of the initial state validation phase. It will be applied for the whole duration of the operation and monitor the subjects' capacity to perform their tasks. It will classify their emotional state and will be able to predict dangerous situations and warn the system's administrator in order to prevent accidents. Changes in physiological features, such as EEG and ECG indicators will be used to classify the subject's emotional state and detect abnormal patterns corresponding to lack of attention, panic, and other basic emotional states that can potentially hinder optimal performance. It is important to note that this mode will only be applied on critical operation scenarios and the subject will always be aware of the monitoring via visual interfaces (e.g., a warning light and a notification). 
TABLE 1: HUMABIO operating modes, corresponding biometric modalities, and identification techniques.

\begin{tabular}{|c|c|c|c|}
\hline Phase & State & Methods & Application \\
\hline \multirow[t]{3}{*}{ Authentication } & Initial & $\begin{array}{l}\text { Password } \\
\text { RFID token } \\
\text { Face recognition } \\
\text { Text dependent voice verifi- } \\
\text { cation } \\
\text { Event related potentials }\end{array}$ & $\begin{array}{l}\text { When the subject logs into } \\
\text { the protected system } \\
\text { Before validation phase }\end{array}$ \\
\hline & Continuous & $\begin{array}{l}\text { EEG baseline } \\
\text { ECG features } \\
\text { Face authentication } \\
\text { Speaker verification (free } \\
\text { speech analysis) }\end{array}$ & $\begin{array}{l}\text { Continuously while the } \\
\text { subject performs his/her } \\
\text { tasks or accesses a protected } \\
\text { resource }\end{array}$ \\
\hline & Nonobtrusive & $\begin{array}{l}\text { RFID token } \\
\text { Face authentication } \\
\text { Gait authentication } \\
\text { ECG features }\end{array}$ & $\begin{array}{l}\text { When the subject accesses a } \\
\text { protected area and is able to } \\
\text { move freely }\end{array}$ \\
\hline $\begin{array}{l}\text { Validation of initial } \\
\text { "nominal" state }\end{array}$ & & $\begin{array}{l}\text { Event related potentials } \\
\text { ECG analysis } \\
\text { Voice analysis } \\
\text { Equilibrium analysis }\end{array}$ & $\begin{array}{l}\text { Before the subject } \\
\text { commences his/her tasks }\end{array}$ \\
\hline Monitoring & & $\begin{array}{l}\text { EEG features } \\
\text { ECG features } \\
\text { Speaker verification (free } \\
\text { speech analysis) }\end{array}$ & $\begin{array}{l}\text { Continuously, while the } \\
\text { subject performs his/her } \\
\text { tasks }\end{array}$ \\
\hline
\end{tabular}

The abnormal states that will be monitored in HUMABIO are the effects of drug and alcohol consumption and sleep deprivation. These conditions were selected because they are some of the major factors that cause operator-related accidents.

\section{ARCHITECTURE}

Modular, open and efficient system architecture has been designed, in order to address the different applications and systems of HUMABIO (see Figure 1).

The design and development of every architectural module takes into account all relevant and important elements, like system requirements, security requirements, risk factors, software issues, communication elements, safety issues (electromagnetic interference and compatibility (EMI/EMC) included), hardware requirements (dimensions, power consumption, etc.), and specific application requirements (e.g., vehicle integration requirements for a transport application). Also issues like the geographical distribution of the system components, data access, data security mechanisms, and compliance with international standards [12] are taken into account.

In order to evaluate the effectiveness of the prototype that integrates all the software and hardware modules and show its modularity and adaptation in versatile scenarios, a series of pilots will be designed and realized.

\section{HUMABIO PILOTS}

\subsection{Pilot plans}

Three applications are considered in order to highlight the modularity of HUMABIO and its adaptability to different application scenarios. In these applications, the physiological and behavioural profiles work either complementary in case one of the two cannot be utilized, acquired, or in parallel, thus strengthening the reliability of the system. Specifically, the applications include the integration of HUMABIO in

(1) a truck, representing in general the transport means environment,

(2) an office environment, for resources protection from unauthorized access and for the evaluation of the system as an emotional state classifier,

(3) an airport, for nonstop and unobtrusive authentication of employees in the controlled area.

Aiming at user's convenience, synergies with undergoing Ambient Intelligence Projects will be pursued. Specifically, the experience from the ASK-IT EU Integrated Project [13] is expected to be transferred to the restricted area pilot, while the possibility of HUMABIO integration in a larger scale AmI environment such as Philips HomeLab Project will be studied in the frame of the office pilot.

Table 2: It correlates the applications to the HUMABIO platform configurations, in order to show the multimodality of the system. 
TABLE 2: HUMABIO operating modes and exploited biometric modalities for each of the pilot scenarios.

(a)

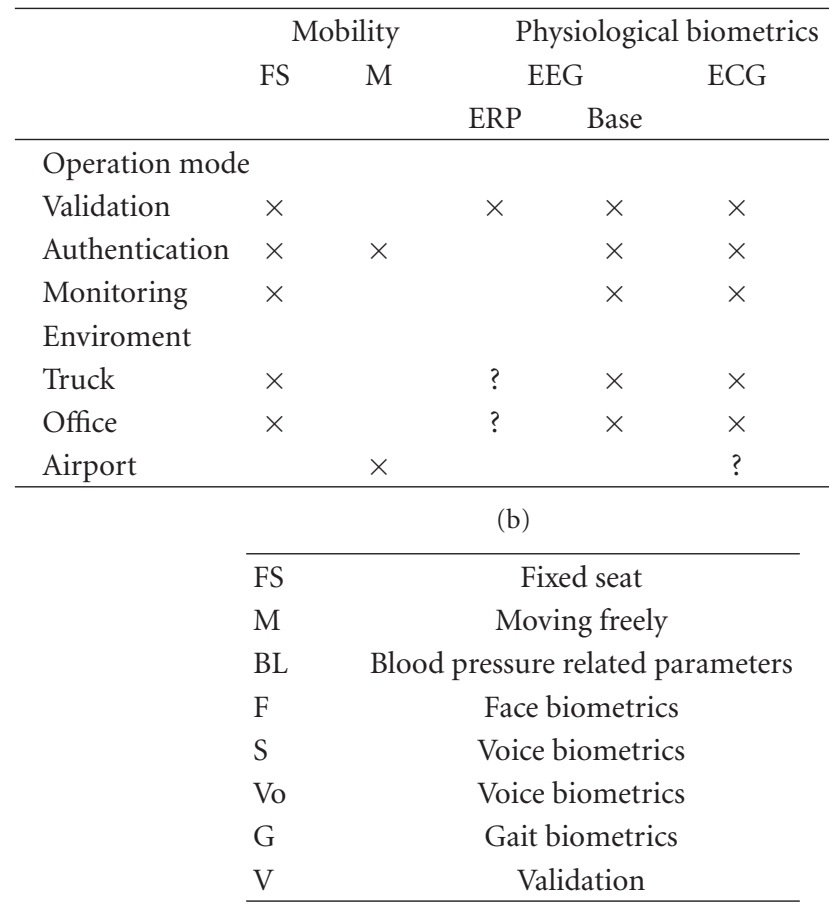

In this paper, the restricted area pilot, which demonstrates the unobtrusiveness of the system, is presented along with some preliminary results for the relevant biometric modalities.

\subsection{Description of restricted area pilot}

The system will be installed in a controlled area in Euroairport in Basel, Switzerland. The aim is to authenticate the identity of authorized employees that can move freely in the area. Depending on the acceptable obtrusiveness level, the appropriate sensor setup will be utilized. Two possible obtrusiveness scenarios are considered depending on the required security level:

(1) the totally unobtrusive scenario, which dictates that the employees will not carry any sensor on them, which in turn means that the physiological profile of the subject will not be available and

(2) the partially obtrusive scenario in which wireless wearable sensors and the utilization of the physiological indicators will be included.

The operational setup is depicted in Figure 2.

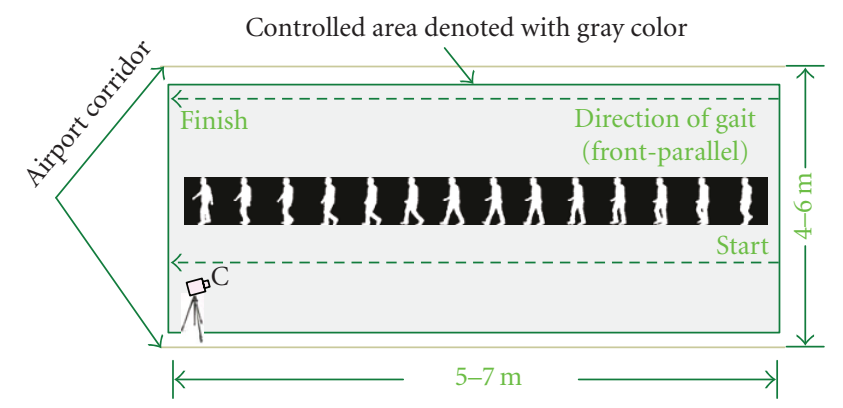

FIGURE 2: Unobtrusive authentication concept for the HUMABIO airport pilot.

\section{Pilot protocol}

The subject will walk along a narrow corridor such as the ones that are usually found in airports. When the subject enters the corridor his (claimed) identity is transmitted wirelessly to the system via an RFID tag. The aim of HUMABIO is to authenticate the claimed identity by the time the subject reaches the end of the corridor.

The corridor's length should be 6 to 7 meters to allow the capturing of sufficient gait information. As the subject walks in the corridor, his gait features are captured by a 


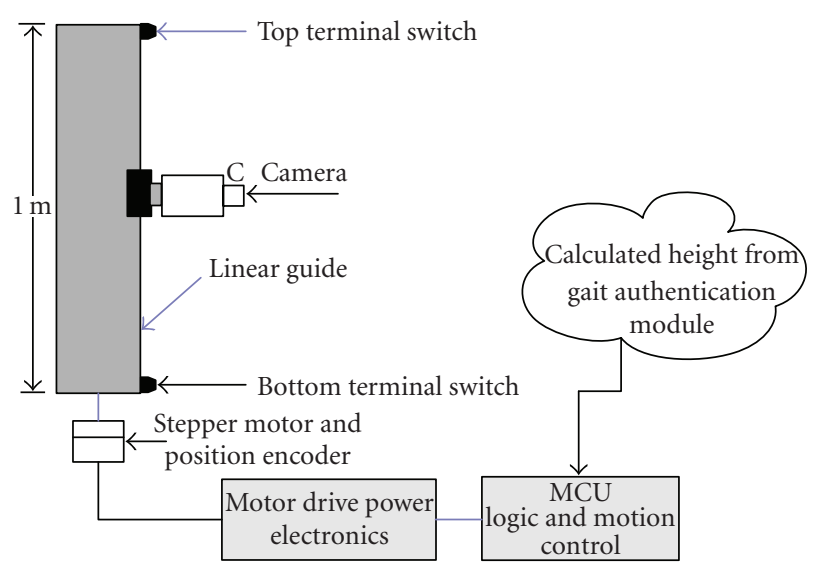

FIgURE 3: Calibration of face recognition camera position based on subject's height information.

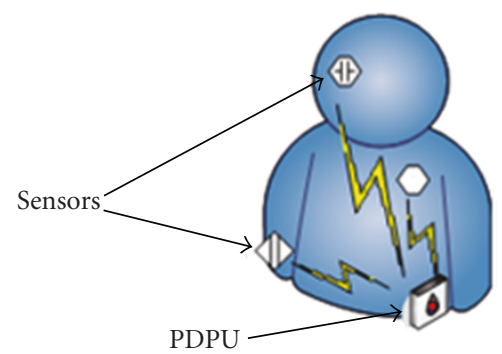

FIGURE 4: Indicative positioning of ENOBIO-based electrodes and the supporting PDPU.

stereoscopic camera and in addition the subject's height is estimated. Height estimation with this method is quite accurate and deviates from the real height by $1 \mathrm{~cm}$ maximum.

Height information is used to calibrate the position of the face recognition camera as shown in Figure 3. Face recognition takes place at the end of the corridor. By the time the subject reaches the camera, its position is already calibrated allowing the unobtrusive face recognition without the need of specific procedures for the collection of the biometric data as it is usually the case with current biometric solutions.

Depending on the required security level more modalities may be utilized to decrease false acceptance ratio (FAR).

The HUMABIO voice recognition module can function in parallel with face recognition. The microphone will be installed at the end of the corridor where face recognition camera is located. The subject will have to pronounce a specific sentence or even talk freely for some seconds, since HUM$\mathrm{ABIO}$ voice recognition modules are able to handle both dictated and free speech.

Physiological signals, namely, EEG and ECG will also be studied for their application potential in this pilot. Preliminary results show that even though EEG using just two electrodes (plus one reference electrode) may yield good authentication rates, this is possible only when a specific procedure is followed so as to avoid the occurrence of artefacts that pollute the necessary for authentication features. These artefacts are caused by muscle activity such as eyelid and eye move-

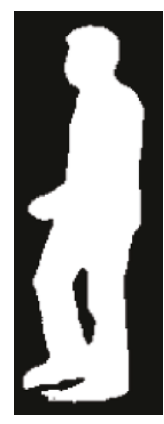

(a)

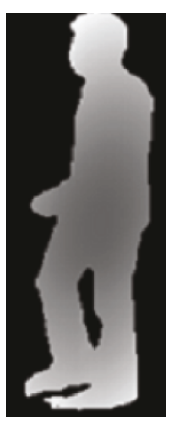

(b)
FIGURE 5: Extracted silhouettes: (a) binary silhouette, (b) geodesic silhouette.

ments, walking, head movement, and so forth. Due to these restrictions EEG is not expected to be applicable in the airport pilot scenario since the person will be mobile and the artefacts from eye activity are inevitable. On the other hand, ECG shows robustness to artefacts and can be acquired by using only one electrode (plus one reference electrode which is common for EEG). ECG's authentication accuracy is comparable to EEG's and more robust due to less interference from muscle activity due to the location of the electrode on the wrist.

\section{Sensors}

The sensors that will be used in the first scenario are RFID tags, a stereoscopic camera for gait recognition and height estimation, a simple camera for face recognition, and possibly a microphone. Since there will be no sensors attached to the subject, the whole process will be transparent and totally unobtrusive.

The sensors that will be used in the second scenario are the ones in the previous scenario with the addition of minimally obtrusive wearable sensors and the personal data processing unit (PDPU). The wearable sensors are electrodes based on the ENOBIO technology [21] that was developed within the SENSATION IP [14]. These electrodes use nanocarbon substrate to stick to the skin without the need of conductive gel. The ECG signal is then transmitted wirelessly to the PDPU for processing and features extraction (see Figure 4). The features are then transmitted to the HUMABIO system for matching with the corresponding templates. The availability of physiological measurements could potentially be used also for the assessment of the subjects' capacity to perform their task.

\section{HUMABIO gait recognition module}

Gait recognition algorithm development uses a novel approach in HUMABIO [20] which involves several stages.

\subsubsection{Binary silhouette extraction}

The walking subject silhouette is extracted from the input image sequence. Initially, the background is estimated using 
a temporal median filter on the image sequence, assuming static background and moving foreground. Next, the silhouettes are extracted by comparing each frame of the sequence with the background. The areas where the difference of their intensity from the background image is larger than a predefined threshold are considered as silhouette areas. Morphological filtering, based on antiextensive connected operators [23], is applied so as to denoise the silhouette sequences. Finally, shadows are removed by analyzing the sequence in the HSV color space [24].

\subsubsection{Generating 3D geodesic silhouettes}

Using the aforementioned techniques, a binary silhouette sequence $\widetilde{B}^{\text {Sil }}$ is generated as illustrated in Figure $5(\mathrm{a})$. In the proposed framework, 2.5D information is available since the gait sequence is captured by a stereoscopic camera. Using Delaunay triangulation on the $2.5 \mathrm{D}$ data, a 3D triangulated hull of the silhouette is generated that is further processed using the proposed 3D Protrusion Transform.

Initially, the triangulated version of the $3 \mathrm{D}$ silhouette is generated. Adjacent pixels of the silhouette are grouped into triangles. Next, the dual graph $G=(V, E)$ of the given mesh is generated [25], where $V$ and $E$ are the dual vertices and edges. A dual vertex is the center of the mass of a triangle and a dual edge links two adjacent triangles. The degree of protrusion for each dual vertex results from

$$
p(\mathbf{u})=\sum_{i=1}^{N} g\left(\mathbf{u}, \mathbf{v}_{i}\right) \cdot \operatorname{area}\left(\mathbf{v}_{i}\right),
$$

where $p(\mathbf{u})$ is the protrusion degree of dual vertex $\mathbf{u}, g\left(\mathbf{u}, \mathbf{v}_{i}\right)$ is the geodesic distance of $\mathbf{u}$ from dual vertex $\mathbf{v} i{ }_{i}$, and area $(\mathbf{v}$ ${ }_{i}$ ) is the area of triangle that corresponds to the dual vertex $\mathbf{v}$

Let us define $G_{k}^{\prime \text { Sil }}(\mathbf{u})$, a function that refers to the dual vertices, to be given by

$$
G_{k}^{\prime S i l}(\mathbf{u})=p(\mathbf{u}) \cdot \widetilde{B}_{k}^{\text {Sil }}(\mathbf{u}) .
$$

The 3D PT for the silhouette image, denoted as $G_{k}^{\mathrm{Sil}}(x, y)$, is simply a weighted average of the dual vertices that are adjacent to the corresponding pixel $(x, y)$, that is,

$$
\begin{aligned}
& G_{k}^{\text {Sil }}(x, y)=\sum_{i=1}^{8} G_{k}^{\prime S i l}(\mathbf{u}) \cdot w(x, y, \mathbf{u}), \\
& \hat{G}_{k}^{\text {Sil }}(x, y)=m+G_{k}^{\text {Sil }}(x, y) \cdot(255-m),
\end{aligned}
$$

where $i=1, \ldots, 8$ denotes the number of adjacent pixels $(x, y)$ to be weighted, $w(x, y, \mathbf{u})$ is the weighting function, and $\hat{G}_{k}^{\text {Sil }}(x, y)$ represents the geodesic silhouette image at frame $k$, as illustrated in Figure 5(b), which takes values in the interval of $[m, 255]$. The higher the intensity value of a pixel in Figure 5(b), the higher its protrusion degree. In the proposed approach, $m$ was selected to be equal to 60 .

\subsubsection{Gait sequence feature extraction}

In the present work, the use of descriptors based on the weighted Krawtchouk moments is proposed. In all cases, the input to the feature extraction system is assumed to be either the binary silhouettes $\left(\widetilde{B}_{k}^{\text {Sil }}\right)$ or the $3 \mathrm{D}$-distributed silhouettes -Sil

$\left(G_{k}\right)$ when the 3D PT is used.

For almost all recent approaches on gait analysis, after feature extraction, the original gait sequence cannot be reconstructed. In the suggested approach, the use of a new set of orthogonal moments is proposed based on the discrete classical weighted Krawtchouk polynomials [26]. The orthogonality of the proposed moments assures minimal information redundancy. In most cases, Krawtchouk transform is used to extract local features of images [26]. The Krawtchouk moments $Q_{n m}$ of order $(n+m)$ are computed using the weighted Krawtchouk polynomials for a silhouette image (binary or 3D) with intensity function $\operatorname{Sil}(x, y)$ by [26]

$$
\begin{aligned}
Q_{n m}= & \sum_{x=0}^{N-1} \sum_{y=0}^{M-1} \bar{K}_{n}(x ; p 1, N-1) \\
& * \bar{K}_{m}(y ; p 2, M-1) \cdot \operatorname{Sil}(x, y), \\
\bar{K}_{n}(x ; p, N)= & K_{n}(x ; p, N) \sqrt{\frac{w(x ; p, N)}{\rho(n ; p, N)}},
\end{aligned}
$$

where $\bar{K}_{n}, \bar{K}_{m}$ are the weighted Krawtchouk polynomials, and $(N-1) \times(M-1)$ represents the pixel size of the silhouette image Sil $(x, y)$. A more detailed analysis of Krawtchouk moments and their computational complexity is presented in [26].

Krawtchouk moments can be used to extract local information of the images by varying the parameters $N$ and $M$. Parameter $N$ can be used to increase the extraction of silhouette image in the horizontal axis. Larger $N$ provides more information on the silhouette image in the horizontal axis, whereas the parameter $M$ extracts local informaton of the silhouette image in the vertical axis. For the experiments, values for $N=R / 15$ and $M=C / 3$ were used, where $R$ and $C$ denote the number of rows and columns of the silhouette image, respectively.

Krawtchouk transform is proposed for feature extraction, due to its very high discriminative power. Krawtchouk transformation is scale and rotation dependent. However, silhouette sequences are prescaled and aligned to the center, thus the Krawtchouk transform is unaffected by scaling. Furthermore, the input gait sequences are captured in a near fronto-parallel view and thus rotation does not affect the results of the Krawtchouk transform.

\subsubsection{Signature matching}

The following notations are used in this section: the term gallery is used to refer to the set of reference sequences, whereas the test or unknown sequences to be verified or identified are termed probe sequence. In this study, the gait cycle is detected using a similar approach to [27], using autocorrelation of the input periodic signal. Instead of measuring only the sum of the foreground pixels in a temporal manner, the time series of the width of the silhouette sequence was also 
calculated. Then, the mean value of these signals formed the final gait period of the current gait sequence.

Each probe sequence is initially partitioned into several full gait cycle segments and the distance between each segment and the gallery sequence is computed separately. This approach can be considered as a brute-force attempt to match a pattern of segmented feature vectors (segmentation using gait cycle) by shifting them over a gallery sequence vector. The main purpose of this shifting is to find the minimum distance (or maximum similarity) between the probe and the gallery sequence.

Let $\mathbf{F}_{P, T}, \mathbf{F}_{G, T}$ represent the feature vectors of the probe with $N_{P}$ frames and the gallery sequence with $N_{G}$ frames, respectively, and let $T$ denote the Krawtchouk transform. The probe sequence is partitioned into consecutive subsequences of $T_{P}$ adjacent frames, where $T_{P}$ is the estimated period of the probe sequence. Also, let the $k$ th probe subsequence be denoted as $\mathbf{F}_{(P, T)}^{k}=\left\{\mathbf{F}_{P, T}^{k T_{P}}, \ldots, \mathbf{F}_{P, T}^{(k+1) T_{P}}\right\}$ and let the gallery sequence of $N_{G}$ frames be denoted as $\mathbf{F}_{G, T}=\left\{\mathbf{F}_{G, T}^{1}, \ldots, \mathbf{F}_{G, T}^{N_{G}}\right\}$. Then, the distance metric between the $k$ th subsequence and the gallery sequence is

$$
\begin{array}{r}
\operatorname{Dist}_{T}(k)=\min _{l} \sum_{i=0}^{T_{P}-1} \sqrt{\sum_{x=0}^{S-1}\left(\mathbf{F}_{P, T}^{i+k \cdot T_{P}}(x)-\mathbf{F}_{G, T}^{i+l}(x)\right)^{2}}, \\
k=0, \ldots, m-1,
\end{array}
$$

where $l=0, \ldots, N_{G}-1, S$ denotes the size of a probe/gallery feature vector $\mathbf{F}$, and $m=N_{P} / T_{P}$ represents the number of probe subsequences.

Equation (5) indirectly supposes that the probe and gallery sequences are aligned in phase. After computing all distances between probe segments and gallery sequences of feature vectors, the median, [30] of the distances is taken as the final distance $D_{T}$ (Probe, Gallery) between the probe and the gallery sequence,

$$
D_{T}=\operatorname{Median}\left(\operatorname{Dist}_{T}(1), \ldots, \operatorname{Dist}_{T}(m)\right), \quad m=\frac{N_{P}}{T_{P}},
$$

where $m$ denotes the number of distances calculated between the probe subsequences and the whole gallery sequence. In (6), smaller distance means a closer match between the probe and the gallery sequence.

\subsubsection{Experimental results}

The proposed method was evaluated on the publicly available HumanID "Gait Challenge" dataset.

Since the HumanID "Gait Challenge" dataset includes only monoscopic image sequences, it cannot be used to evaluate the proposed scheme using the 3D PT. However, the Krawtchouk descriptor efficiency on binary silhouettes was evaluated using this database, so as to generate comparative results with state-of-the-art approaches. In an identification scenario, a score vector for a given probe gait sequence is calculated, that contains the distance of the probe sequence from all the gallery sequences that exist in a database. The gallery sequence that exhibits the minimum distance from

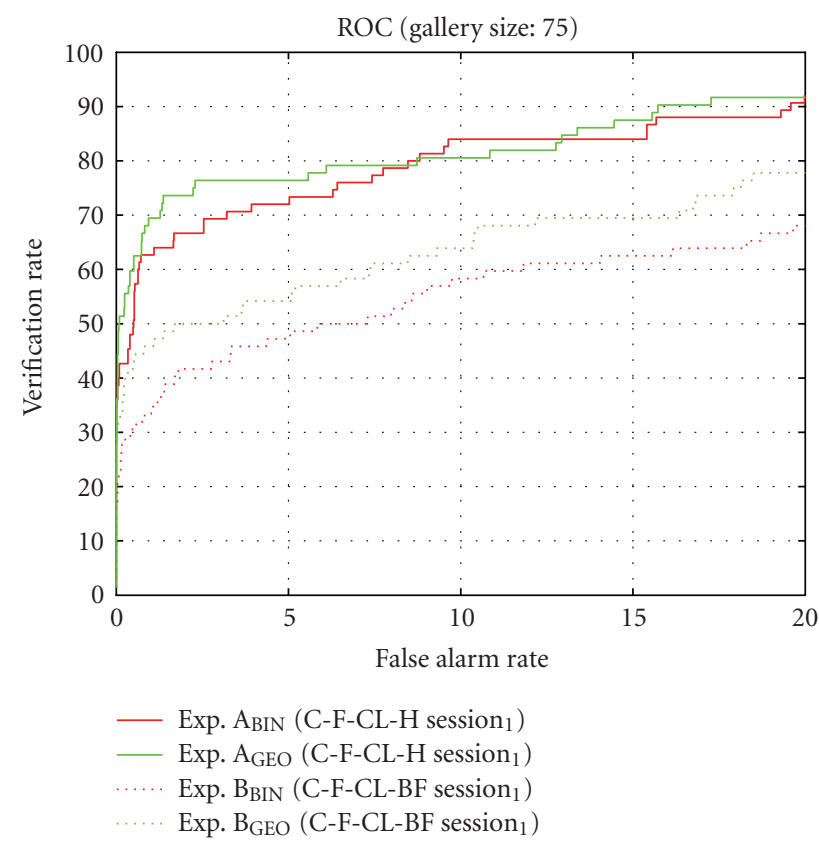

FIGURE 6: Identification rate of the 3D PT method, (3D PT) for two experiments (A, B), compared to the algorithm that uses the Krawtchouk descriptors on binary silhouettes (KW).

the probe sequence is identified as the correspondent sequence to the probe sequence.

In the USF's Gait Challenge Database, the gallery sequences were used as the systems knowledge base and the probe sequences as the ones that should be recognized by comparing their descriptors to the gallery set. The available gallery sequences include $(C, G)$ cement or grass surface, $(A$, B) shoe type A or B, and $(L, R)$ two different view points. In the performed experiments, we used the set GAR as the gallery. The probe set is defined using seven experiments A-G of increasing difficulty. Experiment A differs from the gallery only in terms of the view, B of shoe type, C of both shoe type and view, D of surface, E surface and shoe type, F of surface and viewpoint, and G of all surface, shoe type, and viewpoints.

For evaluation of the proposed approach, cumulative match scores (CMS) are reported at ranks 1 and 5. Rank 1 performance illustrates the probability of correctly identifying subjects in the first place of the ranking score list and the rank 5 illustrates the percentage of correctly identifying subjects in one of the first five places.

Table 3 illustrates rank 1 and 5 results of the proposed approach on binary silhouettes (KR) compared to the CMU [28], LTN-A [29], and BASE approaches [30]. It is obvious that the proposed approach based on Krawtchouk moments performs better in almost all experiments.

3D PT was tested using HUMABIO proprietary gait database consisting of stereoscopic gait sequence recordings from 75 subjects under various conditions. The sequences include (C) normal surface, (CL, PA) shoe type classic or slipper, (BF, NB) carrying a briefcase or not, and $(\mathrm{H})$ when the subject wears a hat. In this paper, two experiments on this 
TABLE 3: Comparative results for the Krawtchouk transform on binary silhouettes (the number of subjects in each set is reported in squared brackets).

\begin{tabular}{lccccccc}
\hline Probe set & \multicolumn{3}{c}{ Rank 1 } & \multicolumn{3}{c}{ Ranks 1-5 } \\
\hline Gallery: GAR & KR & CMU & LTN-A & BASE & KR & CMU & LTN-A \\
\hline A (GAL) [71] & 96 & 87 & 89 & 79 & 100 & 100 & 99 \\
B (GBR) [41] & 85 & 81 & 71 & 66 & 93 & 90 & 81 \\
C (GBL) [41] & 76 & 66 & 56 & 56 & 89 & 83 & 78 \\
D (CAR) [70] & 30 & 21 & 21 & 29 & 63 & 59 & 50 \\
E (CBR) [44] & 27 & 19 & 26 & 24 & 66 & 50 & 57 \\
F (CAL) [70] & 20 & 27 & 15 & 30 & 49 & 53 & 35 \\
G (CBL) [44] & 21 & 23 & 10 & 10 & 48 & 43 & 35 \\
\hline
\end{tabular}

TAble 4: Preliminary performance results for the different modalities that will be utilized in the restricted area pilot.

\begin{tabular}{lll}
\hline $\begin{array}{l}\text { Biometric } \\
\text { modality }\end{array}$ & $\begin{array}{l}\text { Authentication accuracy } \\
\text { range depending on the } \\
\text { experiments (equal error } \\
\text { rate (\%)) }\end{array}$ & Databases used \\
\hline Face [16] & $8-18$ & $\begin{array}{l}\text { ATT and proprietary } \\
\text { databases } \\
\text { YOHO, KING, and } \\
\text { proprietary databases }\end{array}$ \\
ECG [18] & $\sim 3,2$ & \begin{tabular}{l} 
Proprietary \\
\hline
\end{tabular} \\
\hline
\end{tabular}

database are demonstrated. The experiment $\mathrm{A}$ refers to the difference between hat and normal, and the experiment $\mathrm{B}$ refers to the difference between carrying briefcase and normal.

Figure 6 illustrates detailed results on the identification rate of the 3D PT when compared to the algorithm that uses the Krawtchouk descriptors on binary silhouettes. As illustrated, an increased identification rate can be expected when using the 3D PT.

\section{Preliminary authentication results for the rest of the modalities that will be used in the airport scenario}

In Table 3, indicative preliminary results for the modalities that will be used in the airport pilot are presented.

Even though the presented rates are not comparable to the ones found in literature for conventional biometrics such as fingerprint or iris recognition, one must take into account that the approach followed in HUMABIO aims at user convenience and unobtrusiveness. For the achievement of current biometrics' claimed performance, strict protocols are required and also performance during operation in normal conditions deteriorates significantly [22].

Below, the test conditions for the reported results are described for each modality.

\section{Face [16]}

The developers follow the SOA approach that was introduced in [32]. Specifically, three statistical methods are applied to normalized and preprocessed face images represented as high-dimensional pixel arrays to perform classification in lower-dimensional (often) linear subspaces:

(1) the Eigenfaces approach [33], which uses principal component analysis (PCA), a dimensionality reduction method, to extract a number of principal components (the directions of largest variations) from a high dimensional dataset;

(2) the Fisherfaces algorithm [34], based on linear discriminant analysis (LDA) to find the directions in a dataset in which the different classes/individuals are best linearly separable;

(3) the Bayesian face recognition method [35] which computes two linear subspaces: one for intrapersonal variations (or intraclass variance) and another extrapersonal variations (or interclass variance). Classification is performed using maximum a posteriori (MAP) or maximum likelihood (ML) based similarity measure.

Even though the performance of the algorithms developed is comparable to SOA for benchmarking databases, Siemens's research within HUMABIO goes beyond SOA by testing the algorithm in various external light conditions (night, day), subject appearance changes (facial expression, glasses, beard, hairstyle), face pose, facial expression (smile or talk), face appearance changes after a certain time $(>1$ month), various face poses $\left(<15^{\circ}\right.$ in the YES angle, $<10^{\circ}$ in the NO angle), and presence of glasses aiming to develop a really unobtrusive face recognition system that requires no special cooperation from the subject, and making it also suitable for commercial in-vehicle applications.

\section{Voice [17]}

Mel frequency cepstral coefficients (MFCCs) were compared against perceptual linear predictive (PLP) coefficients [31], using a standard GMM configuration. The use of 13 cepstral coefficients against 16 cepstral coefficients was also evaluated and the results revealed that MFCC-13 acoustic features performed better than the rest on both YOHO and KING databases for dictated and free speech correspondingly. The focus of the module development has been put on practical side issues such as the robustness to environment noise, the rejection of unreliable speech samples, the limited amount of enrolment data, and so forth. Several noise models were 
added to examine the robustness of the system in conditions that simulate real application environments. Currently speech recordings that were made within HUMABIO under different conditions (alcohol consumption, drug consumption, or sleep deprivation) are being tested for their impact on the authentication rates.

\section{ECG [18]}

ECG measurement is performed using one electrode located at the wrist for minimal unobtrusiveness (plus one reference electrode). Preliminary results show ECG's authentication potential but studies with more subjects (currently 45 subjects) are being performed to validate the results. From a large set of different features that were studied (HRV related features, geometric features, entropy, fractal dimension, and energy), only the heart beat shape is selected, since it is the feature with the highest discriminative power among subjects. ECG is not robust to motion artefacts; however, its use will be examined in the airport pilot because of the following reasons. The small number of required electrodes and the wrist location, combined with the airport pilot protocol (the subject walks through a corridor for some seconds, so wrist activity is expected to be low) may allow ECG utilization along with the other biometrics. The use of wireless electrodes is expected to reduce motion and interference artefacts as well. Depending on the data analysis after the testing phase and the performance achieved, the final decision regarding the inclusion of ECG in the final HUMABIO prototype for the airport pilot will be taken.

Unimodal biometrics will be fused in order to achieve high authentication rates. In order to develop the fusion algorithms, virtual subjects will be created. Each of these virtual subjects will be the owner of the available modalities. A limitation with this approach is that the maximum number of virtual subjects is equal to the minimum number of subjects recorded for each modality. To overcome this issue, different groups of virtual subjects will be created (using different combinations of subject recordings, or different sessions) and used for testing.

Preliminary support vector machine (SVM) fusion tests with 20 virtual subjects show that $100 \%$ identification accuracy is feasible; however, further testing using larger test populations is necessary and planned for the remaining part of the project.

\section{CONCLUSION}

In this paper, a novel biometrics authentication system is presented. HUMABIO will utilize micro- and nanosensors that are currently under development in the SENSATION IP, aiming primarily at user's convenience and unobtrusiveness. Novel biometric modalities are being studied and used in order to overcome several shortcomings of the current biometrics solutions, mainly, the strict protocols required to be followed by the subjects. HUMABIO among other innovations will support authentication of the individuals in a continuous way and also allow the monitoring of the physiological parameters to ensure the normal state of critical process operators. Its three pilots are designed in such a way as to demonstrate the versatility and extensive modularity of the system and also provide performance evaluation in realistic application scenarios.

\section{REFERENCES}

[1] T. Matsumoto, H. Matsumoto, K. Yamada, and S. Hoshino, "Impact of artificial "gummy" fingers on fingerprint systems," in Optical Security and Counterfeit Deterrence Techniques IV, vol. 4677 of Proceedings of SPIE, pp. 275-289, San Jose, Calif, USA, January 2002.

[2] S. A. C. Schuckers, "Spoofing and anti-spoofing measures," Information Security Technical Report, vol. 7, no. 4, pp. 56-62, 2002.

[3] A. Ross, A. K. Jain, and J.-Z. Qian, "Information fusion in biometrics," in Proceedings of the 3rd International Conference on Audio- and Video-Based Biometric Person Authentication (AVBPA '01), pp. 354-359, Halmstad, Sweden, June 2001.

[4] M. Indovina, U. Uludag, R. Snelick, A. Mink, and A. K. Jain, "Multimodal biometric authentication methods: a COTS approach," in Proceedings of Workshop on Multimodal User Authentication (MMUA '03), pp. 99-106, Santa Barbara, Calif, USA, December 2003.

[5] L. Biel, O. Pettersson, L. Philipson, and P. Wide, "ECG analysis: a new approach in human identification," IEEE Transactions on Instrumentation and Measurement, vol. 50, no. 3, pp. 808812, 2001.

[6] R. B. Paranjape, J. Mahovsky, L. Benedicenti, and Z. Koles', "The electroencephalogram as a biometric," in Proceedings of the Canadian Conference on Electrical and Computer Engineering (CCECE '01), vol. 2, pp. 1363-1366, Toronto, Ontario, Canada, May 2001.

[7] M. Poulos, M. Rangoussi, N. Alexandris, and A. Evangelou, "On the use of EEG features towards person identification via neural networks," Medical Informatics and the Internet in Medicine, vol. 26, no. 1, pp. 35-48, 2001.

[8] C. Escera, E. Yago, M. D. Polo, and C. Grau, "The individual replicability of mismatch negativity at short and long interstimulus intervals," Clinical Neurophysiology, vol. 111, no. 3, pp. 546-551, 2000.

[9] E. Pekkonen, T. Rinne, and R. Näätänen, "Variability and replicability of the mismatch negativity," Electroencephalography and Clinical Neurophysiology, vol. 96, no. 6, pp. 546-554, 1995.

[10] K. H. Kim, S. W. Bang, and S. R. Kim, "Emotion recognition system using short-term monitoring of physiological signals," Medical and Biological Engineering and Computing, vol. 42, no. 3, pp. 419-427, 2004.

[11] J. L. Andreassi, Psychophysiology: Human Behaviour \& Physiological Response, Lawrence Erlbaum Associates, Mahwah, NJ, USA, 2000.

[12] ISO/IEC JTC 1/SC 37, WG2, SD 19785 CBEFF-Common Biometric Exchange Formats Framework.

[13] ASK-IT IP, http://www.ask-it.org/.

[14] SENSATION IP, http://www.sensation-eu.org/.

[15] HUMABIO STREP, http://www.humabio-eu.org/.

[16] M. Braun and S. Boverie, "Face authentication module," Deliverable D3.1, EU IST HUMABIO Project (IST-2006026990).

[17] C. Ris, "Speaker authentication module," Deliverable D3.2, EU IST HUMABIO Project (IST-2006-026990). 
[18] C. Graff, M. Caparrini, et al., "Physiological signals as potential measures of individual biometric characteristics and recommendations for system development," Deliverable D2.1, EU IST HUMABIO Project (IST-2006-026990).

[19] D. Ioannidis, D. Tzovaras, and Y. Damousis, "Gait authentication module," Deliverable D3.3, EU IST HUMABIO Project (IST-2006-026990).

[20] D. Ioannidis, D. Tzovaras, I. G. Damousis, S. Argyropoulos, and K. Moustakas, "Gait recognition using compact feature extraction transforms and depth information," IEEE Transactions on Information Forensics and Security, vol. 2, no. 3, part 2, pp. 623-630, 2007.

[21] G. Ruffini, S. Dunne, E. Farrés, et al., "ENOBIO—first tests of a dry electrophysiology electrode using carbon nanotubes," in Proceedings of the 28th Annual International Conference of the IEEE Engineering in Medicine and Biology Society (EMBS '06), pp. 1826-1829, New York, NY, USA, August 2006.

[22] A. K. Jain, A. Ross, and S. Pankanti, "Biometrics: a tool for information security," IEEE Transactions on Information Forensics and Security, vol. 1, no. 2, pp. 125-143, 2006.

[23] P. Salembier and F. Marqués, "Region-based representations of image and video: segmentation tools for multimedia services," IEEE Transactions on Circuits and Systems for Video Technology, vol. 9, no. 8, pp. 1147-1169, 1999.

[24] R. Cucchiara, C. Grana, M. Piccardi, A. Prati, and S. Sirotti, "Improving shadow suppression in moving object detection with HSV color information," in Proceedings of IEEE Intelligent Transportation Systems (ITSC '01), pp. 334-339, Oakland, Calif, USA, August 2001.

[25] H.-Y. S. Lin, H.-Y. M. Liao, and J.-C. Lin, "Visual salienceguided mesh decomposition," in Proceedings of the 6th IEEE Workshop on Multimedia Signal Processing (MMSP '04), pp. 331-334, Siena, Italy, September-October 2004.

[26] P.-T. Yap, R. Paramesran, and S.-H. Ong, "Image analysis by Krawtchouk moments," IEEE Transactions on Image Processing, vol. 12, no. 11, pp. 1367-1377, 2003.

[27] N. V. Boulgouris, K. N. Plataniotis, and D. Hatzinakos, "Gait recognition using dynamic time warping," in Proceedings of the 6th IEEE Workshop on Multimedia Signal Processing (MMSP '04), pp. 263-266, Siena, Italy, September-October 2004.

[28] R. Collins, R. Gross, and J. Shi, "Silhouette-based human identification from body shape and gait," in Proceedings of the 5th IEEE International Conference on Automatic Face and Gesture Recognition (AFGR '02), pp. 351-356, Washington, DC, USA, May 2002.

[29] N. V. Boulgouris, K. N. Plataniotis, and D. Hatzinakos, "Gait recognition using linear time normalization," Pattern Recognition, vol. 39, no. 5, pp. 969-979, 2006.

[30] S. Sarkar, P. J. Phillips, Z. Liu, I. Robledo-Vega, P. Grother, and K. W. Bowyer, "The humanID gait challenge problem: data sets, performance, and analysis," IEEE Transactions on Pattern Analysis and Machine Intelligence, vol. 27, no. 2, pp. 162-177, 2005.

[31] F. Bimbot, J.-F. Bonastre, C. Fredouille, et al., "A tutorial on text-independent speaker verification," EURASIP Journal on Applied Signal Processing, vol. 2004, no. 4, pp. 430-451, 2004.

[32] X. Wang and X. Tang, "A unified framework for subspace face recognition," IEEE Transactions on Pattern Analysis and Machine Intelligence, vol. 26, no. 9, pp. 1222-1228, 2004.

[33] M. Turk and A. Pentland, "Eigenfaces for recognition," Journal of Cognitive Neuroscience, vol. 3, no. 1, pp. 71-86, 1991.

[34] P. N. Belhumeur, J. P. Hespanha, and D. J. Kriegman, "Eigenfaces vs. Fisherfaces: recognition using class specific linear projection," in Proceedings of the 4th European Conference on Com- puter Vision (ECCV '96), vol. 1, pp. 43-58, Cambridge, UK, April 1996.

[35] B. Moghaddam, T. Jebara, and A. Pentland, "Bayesian face recognition," Pattern Recognition, vol. 33, no. 11, pp. 1771$1782,2000$. 